\title{
Tecnologia EyA: Uma Ferramenta para Produção e Difusão Automatizada de Aulas Digitais na Web
}

\author{
Waldir Roque \\ Instituto de Matemática, UFRGS \\ 91509-900 - Porto Alegre, RS, Brazil \\ roque@mat.ufrgs.br \\ e \\ Enrique Canessa, Marco Zennaro, Carlo Fonda \\ Science Dissemination Unit, ICTP \\ Strada Costiera 11, 34014 - Trieste, Italy \\ sdu@ictp.it
}

\begin{abstract}
Resumo
Neste artigo apresentamos a tecnologia EyA desenvolvida para produção automática em larga escala de conteúdos de apresentações como aulas elou conferências, na forma de áudio e vídeo, sincronizado com a projeção de slides ou uso do tradicional quadronegro e de outros dispositivos utilizados para ensino. Discutiremos vários aspectos relacionados ao desenvolvimento do hardware e software necessários para a gravação de aulas digitais e difusão automática na Internet com a EyA. A tecnologia EyA é de baixo custo e a sua mais recente versão openEyA segue a política da iniciativa de acesso aberto para disseminação da ciência.
\end{abstract}

Palavras-chave: Aula digital, educação a distância, difusão na Web, tecnologia EyA.

\section{EyA Technology: A Tool for Automated Production and Webcasting of Digital Lectures}

\begin{abstract}
In this paper we present the EyA technology developed for the massive and automated production of lectures and/or conferences in the form of video and audio recordings plus slides synchronized, using traditional chalkboards as found in classrooms and/or any digital presentation. We discuss several aspects related to the hardware and software needed to automate the recordings and its internet webcasting with EyA. The EyA technology is low-cost and its most recent version openEyA follows the open access movement for science dissemination.
\end{abstract}

Keywords: Digital lectures, distance learning, webcasting, EyA technology. 


\section{Visão geral}

A tecnologia da informação e comunicação vem proporcionando enormes avanços em diversas áreas. Em particular, podemos observar recentes avanços na forma e meios disponíveis para a educação e ciência, e entre estes meios podemos citar a utilização da internet para difusão de material composto de áudio e vídeo, e teleconferências via Web (Erol and Li, 2005).

No contexto da Educação a Distância $(\mathrm{EaD})$, bastante progresso já tem sido alcançado; mesmo assim, ainda há muito que ser feito para termos um processo realmente adequado para a $\mathrm{EaD}$ (Friedland et al., 2007). Em geral, a $\mathrm{EaD}$ pode ser implementada de várias formas utilizando várias mídias individualmente ou de forma integrada. Segundo Rollins e Almeroth (Rollins and Almeroth, 2004), os processos envolvidos na $\mathrm{EaD}$ podem ser modelados em quatro fases. A primeira fase está relacionada à criação de um ambiente próprio para facilitação de apresentações multimídia, isto é, a criação de uma sala estruturada, denominada "sala de aula digital". A segunda fase corresponde à estrutura de suporte à produção e difusão unidirecional na Web, dos conteúdos gerados nas salas de aulas digitais, ou seja das "aulas digitais", as quais podem ser transmitidas para um ou mais locais remotos. A terceira fase diz respeito ao suporte à colaboração remota entre "professor-aluno", e finalmente, a quarta fase corresponde ao suporte para o arquivamento dos conteúdos para acesso futuro.

Os processos mais sofisticados da EaD requerem que as aulas digitais sejam transmitidas on-line e em tempo real, com colaboração remota. Para que se tenha tudo isso disponível, se faz necessário a construção de sistemas de captura em vídeo da apresentação multimídia, a integração com a Internet para difusão do conteúdo e a construção de ambientes especiais, quer coletivos quer individuais, para que a audiência possa interagir remotamente com o palestrante. Cada uma dessas fases demanda, por si só, o desenvolvimento de ferramentas e a integração de tecnologias. A criação de salas para a produção de "aulas-digitais" tem um custo elevado e, basicamente, a mesma estrutura é necessária para que a audiência tenha condição de colaboração remota. Por isso, uma das alternativas encontradas para viabilizar a EaD com diminuição de custos é a produção de "aulas digitais" e a sua difusão unidirecional (one-way webcasting) que pode ser em tempo real, ou quase em tempo real, ou apenas por meio da disponibilização dos conteúdos em arquivos, de modo que os interessados possam baixá-los para suas máquinas localmente ou mesmo ter acesso através do fluxo de dados de vídeo (video streaming). A interação nestes casos pode ser obtida pela integração da difusão unidirecional na Web com um Sistema de Gerenciamento de Conteúdos para $e$ Learning proporcionando, por exemplo, a possibilidade de estudantes e professores trocarem notas, idéias, comentários, trabalhos de casa, etc.

Em geral, as aulas ministradas em uma sala de aula comum, não estruturada, são, muitas vezes, bem mais complexas do que as apresentações de conferências ou de seminários. As aulas geralmente incluem o uso simultâneo do tradicional quadro-negro ou quadro-branco e/ou de outros dispositivos como retro-projetores, projeções de arquivos do tipo PPT ou PDF via datashow, ou ainda a exposição direta de objetos (aulas práticas em medicina, por exemplo). Nas áreas de ciências exatas, é bastante comum que as aulas sejam ministradas em laboratórios computacionais, com a utilização de sistemas computacionais e exposição de dados e gráficos em telas. Todos estes dispositivos devem ser levados em consideração quando da criação e publicação do conteúdo das aulas digitais, quer on-line quer off-line, com a finalidade de reconstituir o mais fidedignamente possível o próprio ambiente de ensino existente na sala de aula para os usuários remotos. 
Embora algumas iniciativas já tenham sido propostas (Mukhopadhyay and Smith, 1999), este processo de integração ainda representa um desafio tecnológico. Para a produção e disponibilização de difusão unidirecional na Web aberta, é necessário se adotar aplicações que sejam compatíveis com uma gama de dispositivos que mantenham a qualidade de vídeo e áudio a mais elevada possível, mantendo o tamanho de todos os arquivos sincronizados (vídeo, áudio, slides, etc) o menor possível e considerando, também, a possibilidade de se resgatar informações do quadro-negro de forma automática sem qualquer necessidade de intervenção humana dedicada para tal.

Como o custo financeiro de produção deste tipo de conteúdo ainda é elevado, é também muito importante que toda a cadeia produtiva deste processo seja automatizado, evitando ao máximo os procedimentos manuais de configuração de ambientes, edição e pós-processamento. Isto se torna ainda mais relevante quando se deseja realizar uma produção simultânea de aulas digitais originadas em diferentes salas, como ocorre em um campus universitário onde há muitos eventos.

No mundo atual, há uma demanda para a formação e qualificação de recursos humanos, e uma das políticas que vem sendo adotada é promover meios para ampliar a EaD. No Brasil, temos visto um grande interesse governamental para promoção e ampliação da EaD. Uma das mais importantes necessidades para utilização da EaD é a produção de material de bom nível didático-científico e técnico para ensino via internet. A geração de conteúdo escrito não requer muita sofisticação tecnológica, porém a produção de conteúdo audiovisual requer a integração de várias tecnologias.

Neste artigo apresentamos a tecnologia EyA, desenvolvida para a produção em larga escala de aulas e/ou conferências - na forma de gravação de vídeo e áudio sincronizados com a projeção de slides, gerados a partir da utilização do tradicional giz e quadro-negro encontrados em salas de aula ou qualquer forma de apresentação digital. A proposta da tecnologia EyA é possibilitar meios concretos para a produção automatizada de aulas digitais, observando o bom nível da qualidade técnica, com baixo custo de equipamentos e sistema operacional, procurando mantê-las bem próxima da realidade de uma aula tradicional. Na seção 2 discutimos alguns aspectos da tecnologia EyA e apresentamos alguns exemplos da EyA em ação e na seção 3 finalizamos com alguns comentários, conclusões e perspectivas de desenvolvimento da EyA e suas aplicações.

\section{A Tecnologia EyA}

\subsection{Motivação}

O International Center for Theoretical Physics (ICTP), localizado em Trieste, na Itália, é uma instituição pertencente à UNESCO, criada com o objetivo desenvolver atividades de pesquisa e ensino nas áreas de física e matemática, com ênfase particularmente direcionada para promover a difusão do conhecimento nestas áreas para os países em desenvolvimento. No ICTP são realizados, anualmente, mais de 50 eventos (veja lista em www.ictp.it) como conferências e workshops internacionais, além de seminários e palestras de pesquisadores visitantes. Além disso, o ICTP oferece cursos de pré-doutorado regulares e outros programas de formação.

Com o objetivo de ampliar a audiência dos conteúdos produzidos nos diversos eventos e cursos do ICTP, o grupo de pesquisa da Science Dissemination Unit (SDU) do ICTP realizou um levantamento em 2006 sobre o custo médio de produção de material audiovisual na Itália e Europa, visando ampliar a difusão do conhecimento produzido naquele centro. $\mathrm{O}$ valor apresentado era e ainda é muito alto (um custo de mais de 200 
euros por hora de gravação), o que inviabilizava totalmente a produção de "aulas digitais". Assim, com a finalidade de produzir conteúdos audiovisuais das várias atividades realizadas no ICTP, observando características como:

- Alta qualidade

- Sem (ou com mínima) intervenção humana

- Método não invasivo (captura passiva)

- Baixo custo do equipamento e produção

- Serviço contínuo com pouca manutenção

- Características de baixas-frequências (disponibilização em outras mídias)

- Compatibilidade com todos os web browsers e sistemas operacionais

a SDU desenvolveu a tecnologia EyA, para produção, arquivamento e difusão de apresentações que utilizam outros dispositivos como projetores de imagens de tela de computador (slides PPT, PDF, etc), além da velha e tradicional forma de aula utilizando o giz (canetas) e o quadro-negro (quadro-branco), tudo isso sem qualquer intervenção humana.

Os principais problemas enfrentados no desenvolvimento de uma ferramenta para webcasting de aulas digitais são: as salas de aula não são estruturadas, há vários dispositivos multimídia que podem ser utilizados, inclusive simultaneamente, e nem todos os dispositivos seguem uma única linha de tempo, em outras palavras há a necessidade de sincronização.

\subsection{O Sistema EyA}

A tecnologia EyA, batizada como acrônimo de "Enhance your Audience" ("Amplie sua Audiência"), é constituída pela integração de hardware, software e internet, para a produção, arquivamento e difusão na Web de conteúdos de áudio e vídeo digitais, sincronizados com a projeção de slides, cujo processo de produção e publicação na internet é totalmente automatizado (Canessa et al., 2007a, 2007b).

A EyA procura simplificar um dos grandes problemas relacionados a $\mathrm{EaD}$ que é a criação de salas especiais estruturadas para a produção de aulas digitais (Rollins and Almeroth, 2002), pois esta pode ser adaptada a qualquer sala. A produção de aulas digitais com EyA é realizada gravando-se as imagens e o áudio de uma aula ou conferência qualquer, em blocos de 1 hora, utilizando-se um computador, denominado "produtor", o qual inclui uma câmara de vídeo do tipo webcam e um microfone USB fixo na sala. Com uma câmara digital de alta resolução (sete megapixels ou superior), fotos são realizadas a cada 15 segundos, da região da sala onde se encontra o palestrante, o quadro-negro e telas para a projeção de slides. Atualmente as posições das câmaras de vídeo e fotos são fixas não havendo rastreamento para acompanhar o palestrante. As fotos são controladas por um programa computacional e são imediatamente baixadas da câmara para o computador produtor via USB. O programa compara a nova imagem gerada com a imagem da foto anterior e, quando estas são iguais, o sistema deleta a imagem mais recente com a finalidade de reduzir o custo de espaço físico para armazenamento e também do processo de transferência da imagem. Este processo de transferência da foto leva poucos segundos, o que impossibilita que a câmara realize uma nova foto durante este intervalo de tempo; esta é uma restrição imposta pela taxa de transmissão da imagem da câmera para o computador, denominado "master", que sincroniza áudio-vídeo-foto (a experiência evidencia que fotos tomadas a cada 15 segundos permitem um intervalo adequado para o período de 
refresh na Web). Ao final da gravação, ainda visando diminuir o tamanho dos arquivos de gravação, fotos repetidas ou com pequenas modificações são descartadas automaticamente.

Uma coleção de dados é classificada como temporal (T-Dados) quando esta contém informação temporal, caso contrário é dita atemporal (U-Dados). A captura de imagens de vídeo é um exemplo de T-Dados, já que cada quadro possui uma referência única temporal. Por outro lado, uma foto, a projeção de um slide ou mesmo quando o professor escreve algo no quadro-negro, são exemplos de U-Dados. Desta forma, a produção de aulas digitais deve levar em consideração o tipo do dado e procurar sincronizá-los.

O sistema EyA sincroniza automaticamente as imagens, realizadas pela câmara, com a seqüência de imagens de vídeo/áudio. Todas as fotos, juntamente com o vídeo e as informações sobre a sincronização, são transferidas pela rede, em um arquivo TAR, para um servidor dedicado, denominado "mestre". O servidor mestre expande o arquivo TAR recebido das várias salas onde estão sendo realizadas aulas ou conferências, as quais estão sendo produzidas como aulas digitais, com todas as especificações de salas, períodos de gravação etc, e as põe em uma fila para o pós-processamento. As imagens de vídeo são codificadas no formato ".mov" e podem ser visualizadas utilizando o QuickTime player ou no formato Flash (após uma conversão off-line). Esta transferência é realizada a cada uma hora de gravação e pode ocorrer simultaneamente quando o computador produtor já está realizando as gravações do horário seguinte.

Após a sincronização, o servidor mestre publica os dados de todas as informações relevantes na servidora Web destinatária, para acesso imediato por qualquer usuário. Todo este processo de transferência do produtor para o mestre e a sua publicação no servidor Web é executado durante cerca de 5 minutos. A EyA ainda não possibilita a difusão da aula digital em tempo real, porém todo o conteúdo está disponível logo após o período estipulado de uma hora. Como, em geral, uma aula ou palestra tem duração de uma hora, todo o conteúdo da aula pode ser arquivado para imediata utilização tão logo cada hora de aula tenha se passado.

A Figura 1 ilustra uma sala de aula comum do ICTP com os equipamentos do sistema EyA instalados. Para minimizar problemas de falha em um dos componentes eletrônicos e, por conseguinte a interrupção do processo de gravação da aula digital, o sistema é redundante, como ilustrado na Figura 2. Após o período de um ano de gravações foi observado que o sistema apresentou $99 \%$ de confiabilidade.

No portal do ICTP (www.ictp.tv) estão disponíveis todos os detalhes sobre cada uma das aulas digitais (título da aula digital, sala, horário de início e término, tamanho dos arquivos zipados, etc), podendo-se fazer download das aulas digitais de seu interesse para visualização local ou gravação em $\mathrm{CD}$, ou mesmo assistir a aula digital diretamente do servidor Web (neste caso o usuário deve lembrar que a taxa de transmissão é um fator importante para o processo de fluxo de vídeo - streaming). 


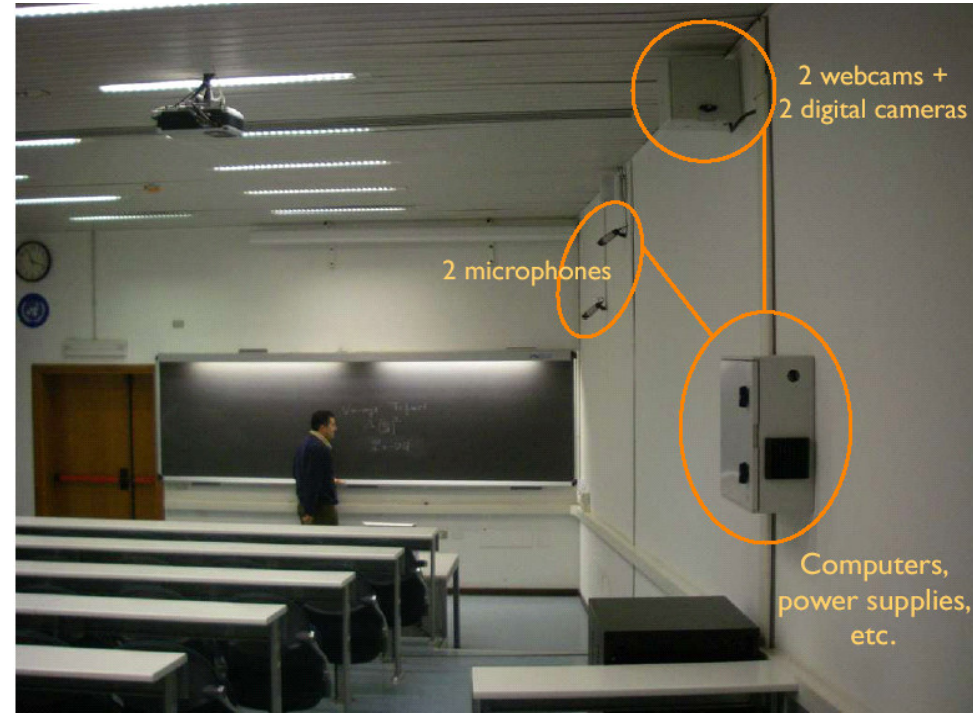

Figura 1 - Ilustração de uma sala contendo a instalação da tecnologia EyA.
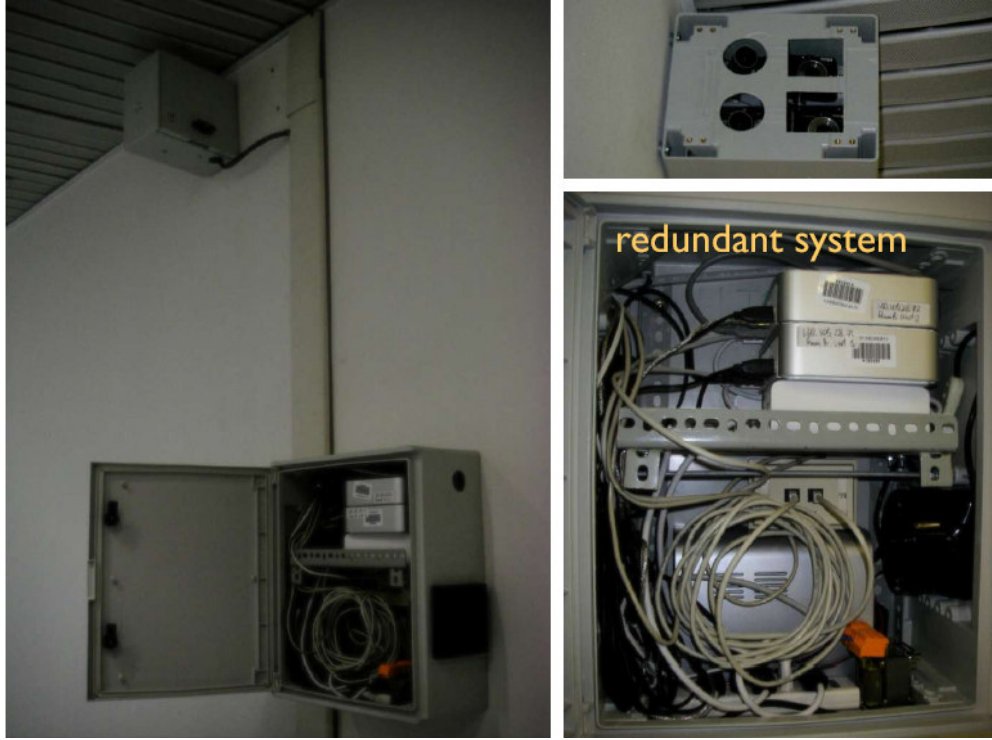

Figura 2 - Ilustração do sistema EyA com redundância.

\subsection{EyA em Ação}

Uma vez disponível a tecnologia EyA a mesma pode ser facilmente instalada qualquer sala para a produção de aulas digitais. No ICTP há atualmente três salas com a tecnologia EyA permanentemente instalada e um equipamento portátil, todos equipamentos com redundância para caso de falhas, além de um computador atuando como master e um servidor Web.

A agenda de controle de funcionamento do sistema EyA é especificada através de uma interface Web gerenciadora a qual, em princípio, pode ser facilmente manipulada por uma secretaria. Nesta são indicados o título do evento, a sala onde este ocorrerá e a hora de início e fim da atividade. Desta forma, todo o ciclo do sistema EyA será automaticamente acionado, indo desde a gravação até a sua publicação na Internet. 
A Figura 3 ilustra como a gravação é visualizada por um usuário remoto. $\mathrm{Na}$ imagem, vemos a apresentação de uma conferência ministrada em um dos laboratórios do ICTP, onde há projeção de slides em tela. Na parte superior esquerda da figura, temos a projeção do vídeo com áudio da aula/conferência e, abaixo desta, vemos a imagem produzida pela câmara digital. Na imagem da câmara há uma janela flutuante, controlada movimentando-se o mouse, que pode navegar sobre todo o quadro da imagem, expandindo a área da mesma sobre a qual a janela flutuante se sobrepõe. A imagem ampliada é vista a direita.

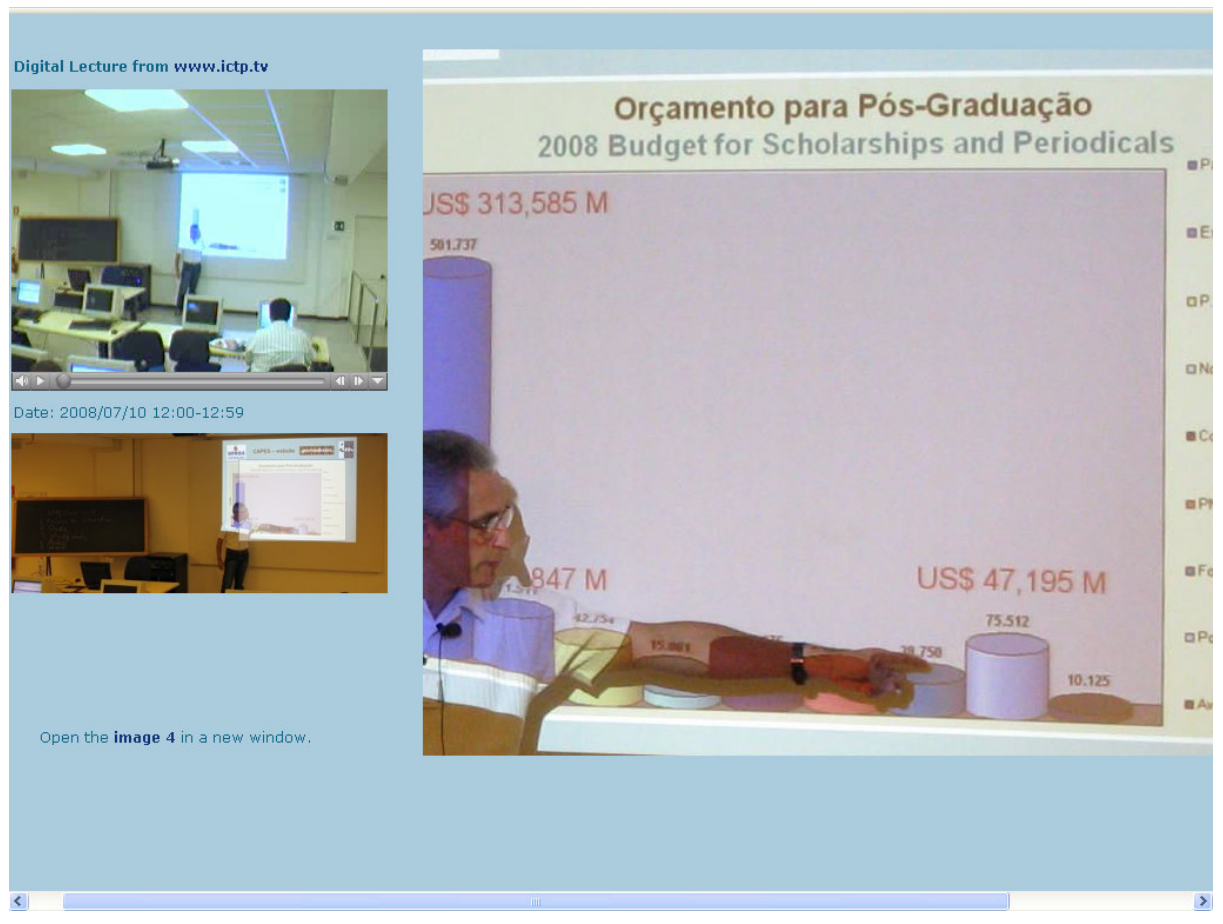

Figura 3 - Imagem de uma tela como vista por um usuário.

Na Figura 4, temos a ilustração de uma sala de aula comum, onde são utilizados simultaneamente a projeção de slides e o tradicional giz e quadro-negro.

\section{Conclusão}

Neste artigo apresentamos uma nova tecnologia para a produção, arquivamento e difusão na Web de aulas digitais. Esta tecnologia, denominada EyA - Enhance your Audience -, é capaz de produzir conteúdos digitais de aulas e/ou conferências de qualidade e com baixo custo, uma vez que todo ciclo de produção indo desde a gravação até a sua publicação na Internet é totalmente automático.

A tecnologia EyA já vem sendo testada durante quase dois anos no International Center for Theoretical Physics - ICTP, em Trieste, Itália, tendo produzido mais de 2.600 horas de aulas digitais entre cursos e conferências, auxiliando assim na disseminação de conhecimento a baixo custo (www.ictp.tv). Hoje é possível sentarmos em um cybercafé e, por menos de um euro, assistirmos uma aula ou uma conferência (PhysicsWorld, 2007), utilizando o fluxo de vídeo sincronizado nos formatos Flash ou 
QuickTime, ou mesmo simplesmente baixarmos o arquivo zipado para uma mídia qualquer (HD, pendrive ou $\mathrm{CD}$ ) e assistirmos posteriormente em qualquer web-browser.
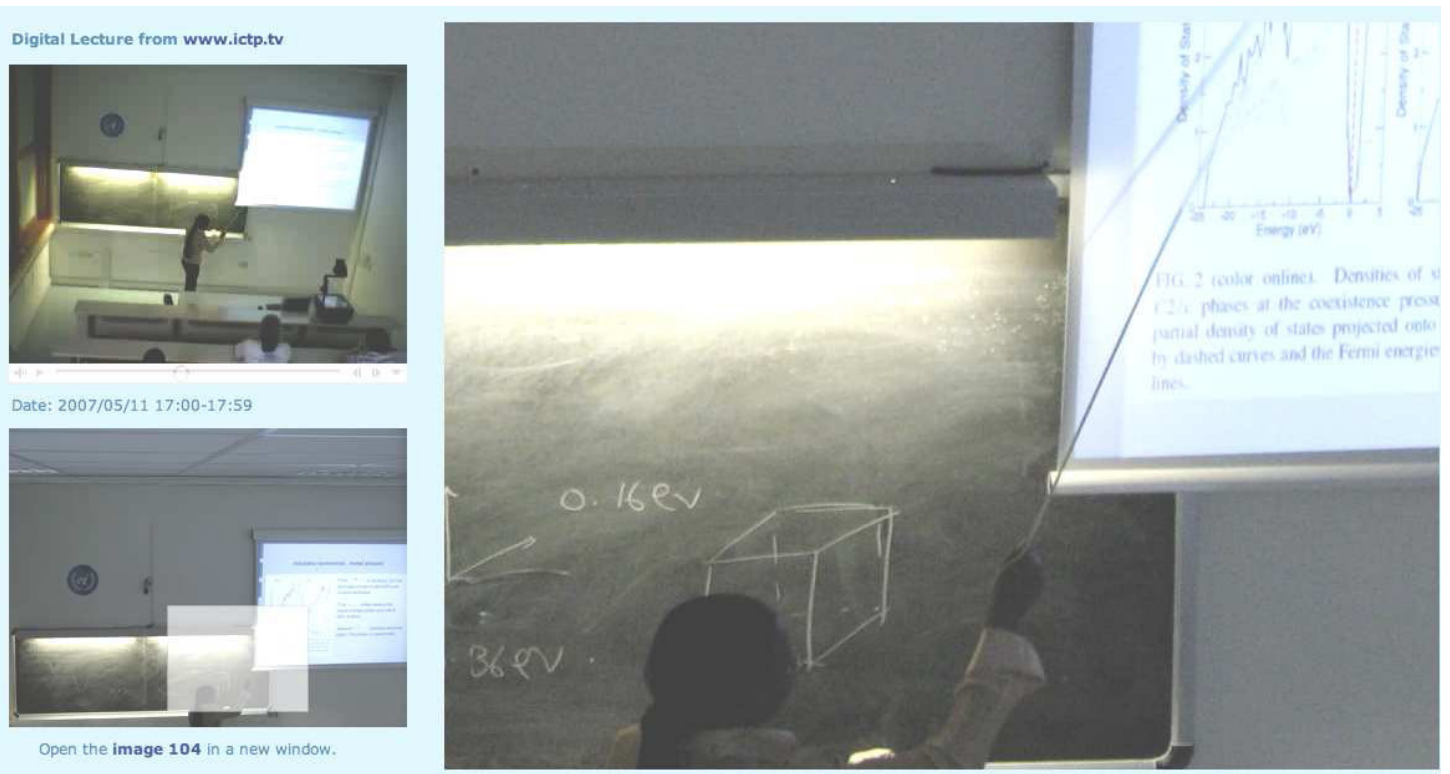

Figura 4 - Aula digital com utilização de slides e quadro-negro.

Inicialmente o sistema EyA foi desenvolvido sobre o sistema operacional da Apple-MacIntosh, que não é um SO livre. Atualmente a SDU está na fase de finalização do desenvolvimento do sistema openEyA (www.openeya.org), que é uma alternativa móvel baseada no sistema operacional Linux (Ubuntu). O custo para a implementação de uma unidade do openEyA é de aproximadamente 700 euros ou menos (em outubro de 2008). Esta unidade móvel funciona como computador "produtor" e "master", diminuindo ainda mais o custo operacional de produção da aula digital.

Para avaliar a importância das aulas digitais das disciplinas oferecidas no ICTP, uma enquete foi realizada entre os estudantes do Diploma Course de 2007-2008 e $97,1 \%$ dos estudantes responderam que as aulas digitais eram muito úteis, particularmente por que permitiam o acesso às disciplinas que não tiveram oportunidade de assistir, por incompatibilidade de horários e pela possibilidade de revisão dos conteúdos das aulas que participaram presencialmente. Na Tabela 1 apresentamos o número de acessos externos ao ICTP, por mês, desde novembro de 2007 a julho de 2008, para fluxo vídeo de aulas digitais apenas das disciplinas oferecidas no Diploma Course do ICTP. Na Tabela 2 apresentamos a média de horas por dia de downloads de aulas digitais destas disciplinas, com aproximadamente 200Mbytes cada.

Tabela 1 - Visitas externas ao ICTP para fluxo de vídeo das disciplinas do Diploma Course.

\begin{tabular}{|c|c|c|c|c|c|c|c|c|}
\hline Nov07 & Dez07 & Jan08 & Fev08 & Mar08 & Abr08 & Mai08 & Jun08 & Jul08 \\
\hline $\mathbf{1 6 8}$ & $\mathbf{5 9 1}$ & $\mathbf{5 9 7}$ & $\mathbf{1 5 1 6}$ & $\mathbf{1 0 3 4}$ & $\mathbf{1 0 1 3}$ & $\mathbf{9 4 2}$ & $\mathbf{1 4 3 6}$ & $\mathbf{1 3 0 4}$ \\
\hline
\end{tabular}


Tabela 2 - Média de horas por dia de download das disciplinas do Diploma Course.

\begin{tabular}{|c|c|c|c|c|c|c|c|c|}
\hline Nov07 & Dez07 & Jan08 & Fev08 & Mar08 & Abr08 & Mai08 & Jun08 & Jul08 \\
\hline $\mathbf{1 9}$ & $\mathbf{1 1 8}$ & $\mathbf{3 5}$ & $\mathbf{9 4}$ & $\mathbf{7 2}$ & $\mathbf{8 0}$ & $\mathbf{7 8}$ & $\mathbf{1 0 0}$ & $\mathbf{7 9}$ \\
\hline
\end{tabular}

Nas universidades federais brasileiras há, em geral, muitas disciplinas que oferecem um grande número de turmas presenciais por semestre. Isto vem demandando a alocação de mais salas de aula e horários distintos, o que muitas vezes dificulta ou mesmo inviabiliza a participação de alunos em tais disciplinas. Uma alternativa para redução do número de turmas presenciais poderia ocorrer selecionando-se grupos de alunos para participarem de aulas digitais (mesmo sem colaboração remota), os quais viriam realizar as provas simultaneamente com os alunos presenciais. Estudantes que apresentam um bom histórico escolar poderiam ter prioridade para participar de disciplinas digitais, uma vez que estes apresentam maior capacidade de aprendizado autônomo, enquanto os estudantes com menor desempenho necessitam de um apoio maior dos professores. É claro que um estudo mais sistemático e detalhado sobre a utilização da EyA como ferramenta de suporte a EaD deve ser conduzido em instituições com um número maior de cursos, de professores e de alunos. Por exemplo, experimentos envolvendo colaboração remota - via Chat em tempo-real, ou via Sistemas de Gerenciamento de Conteúdos como ATutor ou Moodle para interações offline - poderiam ser realizados, pré-agendando-se os horários das aulas virtuais via Web.

Muito ainda há a ser feito visando melhorar a tecnologia EyA, porém com um custo de produção de aulas digitais extremamente baixo e automático, a tecnologia openEyA será, certamente, uma excelente ferramenta para auxílio a programas de Educação a Distância e por conseguinte um forte instrumento de inclusão social.

\section{Referências bibliográficas}

EROL, B. and LI, Y. An Overview of Technologies for e-Meeting and e-Lecture. In: Proceedings of the IEEE ICME, 2005, pp. 6, Amsterdam, The Netherlands, 2005.

FRIEDLAND, G., HÜRST, W. and KNIPPING, L. Educational Multimedia Systems: The Past, the Present, and a Glimpse into the Future. In: Proceedings of the ACM International Workshop on Educational Multimedia and Multimedia Education, Augsburg, Germany, 2007.

ROLLINS, S. and ALMEROTH, K. Lessons Learned Deploying a Digital Classroom, J. of Interactive Learning Research, 15, p.169-185, 2004.

MUKHOPADHYAY, S. and SMITH, B. Passive Capture and Structuring of Lectures. In: Proceedings of $7^{\text {th }}$ Annual ACM International Conference on Multimedia, Orlando, USA: ACM Press, p.477-487, 1999.

CANESSA, E., FONDA, C. and ZENNARO, M., EyA System: Automated AudioVideo-Slide Recordings. In: Proceedings of International Conference on Interactive Computer Aided Learning, September 26 -28, 2007a Villach, Austria.

CANESSA, E., FONDA, C. and ZENNARO, M., Webcasting of Traditional Chalkboard Lectures: The EyA System. European Journal of Open, Distance and e- 
Learning, Issue II, 2007b. Disponível em: <http://www.eurodl.org/>, published 27.09.2007.

ROLLINS, S., ALMEROTH, K. and KLÖSCH, M. Deploying an Infrastructure for Multimedia Enhanced Learning. In: Proceedings of the World Conference on Education Multimedia, Hypermidea \& Telecommunications, Denver, USA, p.16511656, 2002.

PHYSICS WORLD, News \& Analysis, The Video Revolution Made Simple, p. 14, December 2007. 